\title{
ON THE FIXED LOCI OF THE CANONICAL SYSTEMS OVER NORMAL SURFACE SINGULARITIES*
}

\author{
KAZUHIRO $\mathrm{KONNO}^{\dagger}$
}

\begin{abstract}
It is shown that the relative canonical linear system over a normal surface singularity has at most exceptional sets of rational singular points as its fixed part.
\end{abstract}

Key words. Surface singularity, fixed component, reducible curve.

AMS subject classifications. $14 \mathrm{~J} 17$

Introduction. In the study of algebraic surfaces of general type, it is often important to consider the canonical linear system and the rational map associated to it. In this sense, the fixed part of the canonical system can be regarded as the "worst" curve on the surface, and one may naturally ask what is its feature and how to control it. However, not too much is known so far. This is an experiment for a better understanding of the fixed part, and we consider here the local version of the problem.

Let $(V, o)$ be a germ of a normal surface singularity and $\pi: X \rightarrow V$ its minimal resolution. For a line bundle $L$ on $X$ with $H^{0}(X, L) \neq 0$, the fixed part of the linear system $|L|$ is the biggest effective divisor $F$ supported in $\pi^{-1}(o)$ such that the restriction map $H^{0}(X, L) \rightarrow H^{0}(F, L)$ is the zero map. The fixed part of the canonical linear system $\left|K_{X}\right|$ will be sometimes called the canonical fixed part in this paper.

The purpose of the present article is to show the following:

Main Theorem. Let $(V, o)$ be a normal surface singularity and $\pi: X \rightarrow V$ the minimal resolution. If $L$ is a line bundle on $X$ such that $L-K_{X}$ is nef, then the fixed part of $|L|$ supports at most exceptional sets of rational singular points. Furthermore, if $(U, p)$ denotes the rational singular point obtained by contracting a connected component of the fixed part of $|L|$, then the multiplicity $\operatorname{mult}(U, p)$ and the embedding dimension $\operatorname{embdim}(U, p)$ satisfy

$$
\operatorname{mult}(U, p) \leq 2 p_{f}(V, o), \quad \operatorname{embdim}(U, p) \leq 2 p_{f}(V, o)+1
$$

where $p_{f}(V, o)$ denotes the fundamental genus of $(V, o)$, that is, the arithmetic genus of the fundamental cycle on $\pi^{-1}(o)$.

When the fixed part supports exceptional sets of rational double points, we can show that they are necessarily of type A (Corollary 3.3). This suggests that the singular point obtained by contracting a connected component of the canonical fixed part is rather special among rational singular points, though we do not know how to characterize them. We remark that $|L|$ is free from base points if $(V, o)$ itself is a rational singular point (see, Proposition 2.7 for a slightly stronger assertion). So our result applies essentially to singularities "of general type".

On the technical side, one may see that an easy lemma [4, Lemma 2.2.1] plays a very important rôle throughout the paper. In fact, we give in Sect. 1 a decomposition

\footnotetext{
*Received May 20, 2007; accepted for publication May 27, 2008.

${ }^{\dagger}$ Department of Mathematics, Graduate School of Science, Osaka University, Toyonaka, Osaka 560-0043, Japan (konno@math.sci.osaka-u.ac.jp). Partially supported by Grants-in-Aid for Scientific Research (B) (No. 16340008) by Japan Society for the Promotion of Science (JSPS).
} 
of a numerically 1-connected curve, Theorem 1.1, as an application of it. In Sect. 2, we prove the Main Theorem in Theorem 2.4 and Proposition 2.6. Our strategy here is to associate a particular curve, called a loupe, for each fixed component of $|L|$. It is the fundamental cycle on its support, with self-intersection number -1 , containing the fixed component as a non-multiple component. In order to find the loupe, we again use [4, Lemma 2.2.1]. Its decomposition detected by Theorem 1.1 enables us to argue inductively on the number of fixed components. The proof of Proposition 2.7 referred above is also based on [4, Lemma 2.2.1]. In Sect. 3, we state some further properties of the loupes and show Corollary 3.3 as an application. In Sect. 4, we restrict ourselves to (weakly) elliptic singularities [11] in order to clarify, to some extent, how our method relates to Yau's elliptic sequence [13]. When the fixed part corresponds to a rational double point of type A and the biggest loupe contracts to an elliptic singularity, Theorem 4.1 shows that the associated sequence of loupes is nothing more than the elliptic sequence.

In a forthcoming paper, we shall study numerically connected curves and treat the semi-global case, that is, fibers in relatively minimal fibred surfaces.

Acknowledgements. The author thanks Professors Tadashi Tomaru and Margarida Mendes Lopes for their interests and stimulating discussions. He also thanks the referee for his helpful suggestions and careful reading of the manuscript.

1. A decomposition theorem. Let $D=\sum_{i=1}^{N} m_{i} A_{i}$ be a divisor on a nonsingular surface $X$, where $A_{i}$ is a compact irreducible curve and $m_{i} \in \mathbb{Z}$. If all the $m_{i}$ 's are non-negative, $D$ is called effective. For two divisors $D_{1}$ and $D_{2}, D_{2} \preceq D_{1}$ means that $D_{1}-D_{2}$ is effective.

Let $D$ be a non-zero effective divisor. We usually identify it with the corresponding 1-dimensional subscheme of $X$. We put $p_{a}(D)=1-\chi\left(D, \mathcal{O}_{D}\right)$ and call it the arithmetic genus of $D$. By the adjunction formula, we have $2 p_{a}(D)-2=D\left(K_{X}+D\right)$. We say that $D$ is numerically 1 -connected if $D_{1} D_{2}>0$ holds for any effective decomposition $D=D_{1}+D_{2}$ with $0 \prec D_{1}, 0 \prec D_{2}$. A line bundle on $D$ is nef if it is of non-negative degree on any irreducible components. It is well-known that, if $D$ is 1 -connected, we have $h^{0}\left(D, \mathcal{O}_{D}\right)=1$ and for a nef line bundle $L$ we have $h^{0}(D,-L) \neq 0$ if and only if $\mathcal{O}_{D}(L) \simeq \mathcal{O}_{D}$. Furthermore, if we have an effective decomposition $D=D_{1}+D_{2}$ with $D_{1} D_{2}=1$ for a numerically 1-connected divisor $D$, then $D_{1}$ and $D_{2}$ are both numerically 1-connected. For these facts and further properties of 1-connected curves, we refer the readers to [3, Appendix].

Let $L$ be a line bundle on $D$. A point on $D$ is called a base point of the linear system $|L|$ if every section in $H^{0}(D, L)$ vanishes at that point. We denote by $\operatorname{Bs}|L|$ the set of all base points of $|L|$.

The purpose of the section is to show the following theorem. One can find a similar result in $[8$, Theorem 4.1].

THEOREM 1.1. Let $D$ be a numerically 1-connected divisor on a non-singular surface. Assume that an irreducible component $A$ of $D$ is a fixed component of $\left|K_{D}\right|$ and put $n=A(D-A)$. Then $A \simeq \mathbb{P}^{1}$ and, either $D=A$ or there are effective subdivisors $\Gamma_{i}$ of $D(1 \leq i \leq n)$ and a decomposition $D=A+\Gamma_{1}+\cdots+\Gamma_{n}$ enjoying the following properties:

(1) $\Gamma_{i}$ is numerically 1 -connected and $A \Gamma_{i}=1$ holds for each $i \in\{1, \ldots, n\}$.

(2) $A$ is not a component of $\Gamma_{i}$ when $i \geq 2$.

(3) $\mathcal{O}_{\Gamma_{i}+\cdots+\Gamma_{n}}\left(\Gamma_{i-1}\right) \simeq \mathcal{O}_{\Gamma_{i}+\cdots+\Gamma_{n}}$ holds for any $i$ with $2 \leq i \leq n$. 
Proof. We may assume $n>0$, since we clearly have $D=A \simeq \mathbb{P}^{1}$ when $n=0$. We let $D_{1}$ be a minimal effective divisor such that $A \preceq D_{1} \preceq D$ and the restriction map $H^{0}\left(D, K_{D}\right) \rightarrow H^{0}\left(D_{1}, K_{D}\right)$ is surjective.

We first claim that $D_{1} \neq D$. This can be seen as follows. Take any irreducible component $B \preceq D-A$ and consider the cohomology long exact sequence for

$$
0 \rightarrow \mathcal{O}_{B}\left(K_{B}\right) \rightarrow \mathcal{O}_{D}\left(K_{D}\right) \rightarrow \mathcal{O}_{D-B}\left(K_{D}\right) \rightarrow 0 .
$$

Since $H^{1}\left(B, K_{B}\right) \rightarrow H^{1}\left(D, K_{D}\right)$ is dual of the restriction map $H^{0}\left(D, \mathcal{O}_{D}\right) \rightarrow$ $H^{0}\left(B, \mathcal{O}_{B}\right)$, it is an isomorphism, hence $H^{0}\left(D, K_{D}\right) \rightarrow H^{0}\left(D-B, K_{D}\right)$ is surjective. In particular, we can assume that $D_{1} \preceq D-B$ by the minimality of $D_{1}$. Hence $D_{1} \neq D$.

We have $K_{D_{1}}-K_{D}=-\left(D-D_{1}\right)$ on $D_{1}$. Since $D$ is 1 -connected, we have $\left(D-D_{1}\right) D_{1}>0$. In particular, we see that $K_{D_{1}}-K_{D}$ is not nef on $D_{1}$. Then it follows from [4, Lemma 2.2.1] that

(a) $A$ is of multiplicity one in $D_{1}$,

(b) $-\left(D-D_{1}\right)$ is nef on $D_{1}-A$, and

(c) the image of $H^{0}\left(D_{1}, K_{D}\right) \rightarrow H^{0}\left(A, K_{D}\right)$ contains the image of the natural map $H^{0}\left(A, K_{D}-\left(D_{1}-A\right)\right) \hookrightarrow H^{0}\left(A, K_{D}\right)$.

Since we have assumed that $A \subset \mathrm{Bs}\left|K_{D}\right|$, the restriction map $H^{0}\left(D, K_{D}\right) \rightarrow$ $H^{0}\left(A, K_{D}\right)$ is the zero map. Hence so is $H^{0}\left(D_{1}, K_{D}\right) \rightarrow H^{0}\left(A, K_{D}\right)$ by the choice of $D_{1}$. It follows from (c) that $H^{0}\left(A, K_{D}-\left(D_{1}-A\right)\right)=0$. By the adjunction formula, we have $\mathcal{O}_{A}\left(K_{D}-\left(D_{1}-A\right)\right) \simeq \mathcal{O}_{A}\left(K_{A}+\left(D-D_{1}\right)\right)$. Since $-\left(D-D_{1}\right)$ is nef on $D_{1}-A$ by (b), we have $\left(D-D_{1}\right)\left(D_{1}-A\right) \leq 0$. So, $\left(D-D_{1}\right) A>0$, because we have $\left(D-D_{1}\right) D_{1}>0$ by the 1 -connectedness of $D$. Therefore, $\mathcal{O}_{A}\left(K_{D}-\left(D_{1}-A\right)\right)$ is non-special and we get $h^{0}\left(A, K_{D}-\left(D_{1}-A\right)\right)=p_{a}(A)-1+A\left(D-D_{1}\right)$ by the RiemannRoch theorem. Since this has to be zero, we get $p_{a}(A)=0$ and $A\left(D-D_{1}\right)=1$. Then, $\left(D-D_{1}\right)\left(D_{1}-A\right)=0$ and it follows from (b) that $\mathcal{O}_{D_{1}-A}\left(D-D_{1}\right)$ is numerically trivial. We have shown that $A \simeq \mathbb{P}^{1}$ and $D_{1}\left(D-D_{1}\right)=1$. Note that the last equality implies that $D_{1}$ and $D-D_{1}$ are also numerically 1-connected.

Take any point $p \in A$ not lying on $D_{1}-A$. Since $A \subset \mathrm{Bs}\left|K_{D}\right|$, it follows from the cohomology long exact sequence for

$$
0 \rightarrow \mathcal{O}_{D_{1}}\left(K_{D}-p\right) \rightarrow \mathcal{O}_{D_{1}}\left(K_{D}\right) \rightarrow \mathcal{O}_{p} \rightarrow 0
$$

that $H^{0}\left(D_{1}, K_{D}\right) \rightarrow \mathcal{O}_{p}$ is zero, and hence $H^{1}\left(D_{1}, K_{D}-p\right) \neq 0$. By the Serre duality theorem, we get $H^{0}\left(D_{1}, p-\left(D-D_{1}\right)\right) \neq 0$. Since $\mathcal{O}_{D_{1}}\left(p-\left(D-D_{1}\right)\right)$ is numerically trivial and $D_{1}$ is 1-connected, we conclude that $\mathcal{O}_{D_{1}}\left(D-D_{1}\right) \simeq \mathcal{O}_{D_{1}}(p)$. In particular, this shows that $\mathcal{O}_{D_{1}-A}\left(D-D_{1}\right) \simeq \mathcal{O}_{D_{1}-A}$. Furthermore, we have $h^{0}\left(D_{1}, \mathcal{O}_{D_{1}}(p)\right) \geq 2$, since the same argument as above shows $\mathcal{O}_{D_{1}}\left(D-D_{1}\right) \simeq \mathcal{O}_{D_{1}}\left(p^{\prime}\right)$ and, hence, $\mathcal{O}_{D_{1}}(p) \simeq \mathcal{O}_{D_{1}}\left(p^{\prime}\right)$ for any other point $p^{\prime} \in A \backslash \operatorname{Supp}\left(D_{1}-A\right)$. Then we get $h^{0}\left(D_{1}, K_{D_{1}}-p\right)=h^{0}\left(D_{1}, \mathcal{O}_{D_{1}}(p)\right)-2+p_{a}\left(D_{1}\right) \geq p_{a}\left(D_{1}\right)$ by the Riemann-Roch and the Serre duality theorems. On the other hand, we clearly have $h^{0}\left(D_{1}, K_{D_{1}}-p\right) \leq$ $h^{0}\left(D_{1}, K_{D_{1}}\right)=p_{a}\left(D_{1}\right)$. In sum, $h^{0}\left(D_{1}, K_{D_{1}}-p\right)=h^{0}\left(D_{1}, K_{D_{1}}\right)=p_{a}\left(D_{1}\right)$. This shows that $p$ is a base point of $\left|K_{D_{1}}\right|$. Since $p \in A$ is general, we conclude that $A$ is a fixed component of $\left|K_{D_{1}}\right|$.

Since $A \subset \mathrm{Bs}\left|K_{D_{1}}\right|$ and $D_{1}$ is numerically 1-connected, we can repeat the above argument with the pair $\left(D_{1}, A\right)$ instead of $(D, A)$ noting that we have $A\left(D_{1}-A\right)=$ $A(D-A)-A\left(D-D_{1}\right)=n-1$. In this way, we can find a sequence of numerically 1-connected divisors

$$
A=D_{n} \preceq D_{n-1} \preceq \cdots \preceq D_{1} \preceq D_{0}=D
$$


such that $D_{i-1}-D_{i}$ is 1-connected, $\mathcal{O}_{D_{i}-A}\left(D_{i-1}-D_{i}\right) \simeq \mathcal{O}_{D_{i}-A}, A\left(D_{i-1}-D_{i}\right)=1$ and $A \subset \mathrm{Bs}\left|K_{D_{i}}\right|$ for any $i, 1 \leq i \leq n$. If we put $\Gamma_{i}=D_{i-1}-D_{i}$, then $D=$ $A+\Gamma_{1}+\cdots+\Gamma_{n}$. Note that we have $D_{i}-A=\Gamma_{i+1}+\cdots+\Gamma_{n}$. Hence (3) is nothing more than $\mathcal{O}_{D_{i}-A}\left(\Gamma_{i}\right) \simeq \mathcal{O}_{D_{i}-A}$. Since $A$ is of multiplicity one in $D_{1}$ by (a), we get (2).

Remark 1.2. (1) The converse holds in the following sense: If $A \simeq \mathbb{P}^{1}$ and $D$ decomposes as above, then $A \subset \mathrm{Bs}\left|K_{D}\right|$. See, the proof of [8, Theorem 4.1].

(2) The assertion (3) in Theorem 1.1 implies that $\mathcal{O}_{\Gamma_{j}}\left(\Gamma_{i}\right) \simeq \mathcal{O}_{\Gamma_{j}}$ when $i<j$. Therefore, (1) and (3) of Theorem 1.1 (without any assumption regarding $D$ ) imply that either $\operatorname{Supp}\left(\Gamma_{i}\right) \cap \operatorname{Supp}\left(\Gamma_{j}\right)=\emptyset$ or $\Gamma_{j} \preceq \Gamma_{i}$ when $i<j$. Indeed, suppose that $\operatorname{Supp}\left(\Gamma_{i}\right) \cap \operatorname{Supp}\left(\Gamma_{j}\right) \neq \emptyset$. Since $\Gamma_{i} \Gamma_{j}=0, \Gamma_{i}$ and $\Gamma_{j}$ have a common component. Put $B=\operatorname{gcd}\left(\Gamma_{i}, \Gamma_{j}\right), C_{i}=\Gamma_{i}-B$ and $C_{j}=\Gamma_{j}-B$. We have $C_{i} C_{j} \geq 0$, since they have no common components. Assume that $C_{j} \neq 0$. Since $\mathcal{O}_{\Gamma_{j}}\left(\Gamma_{i}\right) \simeq \mathcal{O}_{\Gamma_{j}}$, we have $\mathcal{O}_{C_{j}}\left(\Gamma_{i}\right) \simeq \mathcal{O}_{C_{j}}$ and it follows that $C_{i} C_{j}+B C_{j}=0$. Hence $B C_{j} \leq 0$, which is impossible since $\Gamma_{j}=C_{j}+B$ is 1-connected. Therefore, we have $C_{j}=0$ and $\Gamma_{j}=B \preceq \Gamma_{i}$. This remark will be used in several places in what follows.

Corollary 1.3. Let $D$ and $A$ be as in Theorem 1.1. Assume furthermore that $K_{D}$ is nef. Then $(D-A) A \leq p_{a}(D)$.

Proof. We know $A \simeq \mathbb{P}^{1}$. Put $n=A(D-A)$ and let $D=A+\Gamma_{1}+\cdots+\Gamma_{n}$ be the decomposition as in Theorem 1.1. Then it is easy to see that $p_{a}(D)=p_{a}\left(\Gamma_{1}\right)+$ $\cdots+p_{a}\left(\Gamma_{n}\right)$ by using the numerical properties $A \Gamma_{i}=1$ and $\Gamma_{i} \Gamma_{j}=0$ when $i \neq j$. Since $K_{D}$ is nef, we have $0 \leq\left.\operatorname{deg} K_{D}\right|_{\Gamma_{i}}=\operatorname{deg} K_{\Gamma_{i}}+\left(D-\Gamma_{i}\right) \Gamma_{i}=\operatorname{deg} K_{\Gamma_{i}}+1$. Hence $\operatorname{deg} K_{\Gamma_{i}}=2 p_{a}\left(\Gamma_{i}\right)-2$ is non-negative, implying $p_{a}\left(\Gamma_{i}\right)>0$. Then we get $p_{a}(D) \geq n=A(D-A)$ as desired.

Let $\mathcal{A}=\bigcup_{i=1}^{N} A_{i}$ be a connected bunch of irreducible curves $A_{i}$. The intersection form is negative semi-definite on $\mathcal{A}$ if and only if there exists an effective (non-zero) divisor $Z$ supported on $\mathcal{A}$ such that $-Z$ is nef on $\mathcal{A}$. The smallest curve among such $Z$ 's exists and is called the numerical cycle [10]. When the intersection form is negative definite, it is usually called the fundamental cycle ([1], [2]). It is easy to see that a numerically 1 -connected divisor $Z$ is the numerical cycle (on its support) if $-Z$ is nef on $Z$.

LEMma 1.4. Let $D$ be an effective non-zero divisor on a non-singular surface such that $-D$ is nef on $D$. Assume that $D$ decomposes as $D=A+\Gamma_{1}+\cdots+\Gamma_{n}$ for some positive integer $n$, where $A \prec D$ is an effective divisor, the $\Gamma_{i}$ 's are 1-connected divisors satisfying $A \Gamma_{i}=1$ for $i \geq 1$ and $\mathcal{O}_{\Gamma_{j}}\left(\Gamma_{i}\right)$ is numerically trivial for $i<j$. If $A$ does not have a common component with $\Gamma_{i}$, then $-\Gamma_{i}$ and $-\tilde{\Gamma}_{i}$ are both nef on $\Gamma_{i}$, where $\tilde{\Gamma}_{i}=\sum_{j \geq i, \Gamma_{j} \preceq \Gamma_{i}} \Gamma_{j}$. In particular, $\Gamma_{i}$ is the numerical cycle on its support provided that $A$ does not have a common component with $\Gamma_{i}$.

Proof. We remark that $\Gamma_{i} \Gamma_{j}=0$ and, either $\Gamma_{j} \preceq \Gamma_{i}$ or $\operatorname{Supp}\left(\Gamma_{i}\right) \cap \operatorname{Supp}\left(\Gamma_{j}\right)=\emptyset$ when $i<j$. Let $B$ be an arbitrary irreducible component of $\Gamma_{i}$. Note that any $\Gamma_{j}$ not appearing in $\tilde{\Gamma}_{i}$ is either disjoint from $\Gamma_{i}$ or bigger than $\Gamma_{i}$, and we have $B \Gamma_{j}=0$ for such $\Gamma_{j}$. Then we have $D B=A B+\tilde{\Gamma}_{i} B$. Since $D B \leq 0$ and $A B \geq 0$ by $\operatorname{gcd}\left(A, \Gamma_{i}\right)=0$, we get $\tilde{\Gamma}_{i} B \leq 0$ as wished.

If $B$ is a component of $\Gamma_{j}$ for some $j>i$, then we have $\Gamma_{i} B=0$, because $\mathcal{O}_{\Gamma_{j}}\left(\Gamma_{i}\right)$ is numerically trivial. Hence we may assume that $B$ is not a component of $\Gamma_{j}$ for any 
$j>i$. Then $\Gamma_{j} B \geq 0$ for any $j>i$. We have $0 \geq \tilde{\Gamma}_{i} B=\Gamma_{i} B+B \sum_{j>i, \Gamma_{j}} \preceq \Gamma_{i} \Gamma_{j} \geq$ $\Gamma_{i} B$. Therefore, $-\Gamma_{i}$ is nef on $\Gamma_{i}$. It follows that $\Gamma_{i}$ is the numerical cycle on its support, since $\Gamma_{i}$ is 1 -connected.

Proposition 1.5. Let $Z$ be the fundamental cycle on the exceptional set of a rational normal surface singularity. If $A \prec Z$ is an irreducible component, then $Z$ decomposes as

$$
Z=A+Z_{1}+\cdots+Z_{n}
$$

where $n=A(Z-A)$, each $Z_{i}$ is a 1-connected divisor, $A Z_{i}=1$ and, $\mathcal{O}_{Z_{i}+\cdots+Z_{n}}\left(-Z_{i-1}\right) \simeq \mathcal{O}_{Z_{i}+\cdots+Z_{n}}$ when $2 \leq i \leq n$. Furthermore, $Z_{i}$ is the fundamental cycle on its support when $i \geq 2$, and the same is valid for $Z_{1}$ provided that $A$ is a non-multiple component of $Z$.

Proof. We have $H^{1}\left(Z, \mathcal{O}_{Z}\right)=0$ and $h^{0}\left(Z, \mathcal{O}_{Z}\right)=1$. Hence $Z$ is 1-connected. The restriction map $H^{0}\left(Z, K_{Z}\right) \rightarrow H^{0}\left(A, K_{Z}\right)$ is the zero map for the trivial reason. Hence, by Theorem 1.1, we get the decomposition of $Z$ as wished. The fact that $Z_{i}$ is the fundamental cycle follows from Lemma 1.4, since $A$ is not a component of $Z_{i}$ when $i \geq 2$, by Theorem 1.1, (2).

Let the situation be as above. We remark that, if $A \npreceq Z_{i}$ (which holds at least for $i \geq 2$ ), then $Z_{i}$ has a non-multiple component $A_{i}$ with $A A_{i}=1$ by $A Z_{i}=1$ and, therefore, $Z_{i}$ also decomposes into a sum of $A_{i}$ and several numerically disjoint fundamental cycles similarly as in the statement of Proposition 1.5.

2. Proof of Main Theorem. We return to the situation we are interested in. Let $(V, o)$ be a germ of a normal surface singularity and $\pi: X \rightarrow V$ the minimal resolution. A non-zero effective (integral) divisor on $X$ whose support is contained in $\pi^{-1}(o)$ will be simply called a curve in what follows. Since the intersection form is negative definite on the exceptional set, we have $A^{2}<0$ and $h^{0}\left(A, \mathcal{O}_{A}(A-L)\right)=0$ for any curve $A$ and a nef line bundle $L$. The latter implies, via the Serre duality theorem, that $H^{1}(A, L)=0$ for any line bundle $L$ such that $L-K_{X}$ is nef. We denote by $Z$ the fundamental cycle on $\pi^{-1}(o)$, that is, the smallest curve such that $-Z$ is nef on $\pi^{-1}(o)$. Recall that the restriction map $H^{0}(X, L) \rightarrow H^{0}(Z, L)$ is surjective when $L-K_{X}$ is a nef line bundle on $X$, because we have $H^{1}(X, L-Z)=0$ by the Kodairatype vanishing theorem (see, e.g., [6]). Therefore, when $L-K_{X}$ is nef, an irreducible curve $E$ is contained in $\mathrm{Bs}|L|$ if and only if the restriction map $H^{0}(Z, L) \rightarrow H^{0}(E, L)$ is the zero map.

We sometimes need the following easy lemma (compare this with [7, Lemma 3.2]):

Lemma 2.1. Let $\Delta$ be a curve with $\Delta^{2}=-1$. Then it is numerically 1-connected. If $\Delta^{\prime}$ is another curve with $\left(\Delta^{\prime}\right)^{2}=-1$, then either $\Delta$ and $\Delta^{\prime}$ are disjoint or one is a subcurve of the other.

Proof. Let $\Delta=\Delta_{1}+\Delta_{2}$ be any effective decomposition, that is, a decomposition of $\Delta$ into a sum of two curves $\Delta_{1}, \Delta_{2}$. Then $\Delta^{2}=\Delta_{1}^{2}+\Delta_{2}^{2}+2 \Delta_{1} \Delta_{2}$. Since we have $\Delta_{1}^{2}<0, \Delta_{2}^{2}<0$ and $\Delta^{2}=-1$, we get $\Delta_{1} \Delta_{2}>0$. Therefore, $\Delta$ is numerically 1-connected.

In order to show the second part, we assume that $\operatorname{Supp}(\Delta) \cap \operatorname{Supp}\left(\Delta^{\prime}\right) \neq \emptyset$. Since $0>\left(\Delta+\Delta^{\prime}\right)^{2}=-2+2 \Delta \Delta^{\prime}$, we get $\Delta \Delta^{\prime} \leq 0$. It follows that $\Delta$ and $\Delta^{\prime}$ have a 
common component. Put $C=\operatorname{gcd}\left(\Delta, \Delta^{\prime}\right), \Delta=A+C$ and $\Delta^{\prime}=B+C$. We assume that $A \neq 0, B \neq 0$ and show that this leads us to a contradiction. Since

$$
0>(A+B+C)^{2}=(A+C)^{2}+(B+C)^{2}-C^{2}+2 A B=-2-C^{2}+2 A B
$$

we have $C^{2}>2 A B-2$. It follows from $C^{2}<0$ and $A B \geq 0$ that $C^{2}=-1$ and $A B=0 \mathrm{DThen}$ we get $A C+B C \leq 1$ by

$$
0 \geq \Delta \Delta^{\prime}=C^{2}+(A+B) C+A B=-1+A C+B C .
$$

On the other hand, it follows from $\Delta^{2}=\Delta^{\prime 2}=-1$ that $A^{2}+2 A C=B^{2}+2 B C=0$. Since $A \neq 0$ and $B \neq 0$, we have $A^{2}<0$ and $B^{2}<0$. Hence $A C>0$ and $B C>0$, which contradicts $A C+B C \leq 1$. Therefore, either $A$ or $B$ must be zero.

The following is the heart of our arguments.

Proposition 2.2. Let $L$ be a line bundle on $Z$ such that $L-K_{X}$ is nef. Let $E$ be an irreducible curve contained in $\mathrm{Bs}|L|$. Then $E \simeq \mathbb{P}^{1}$ and there exists the smallest reducible subcurve $\Delta=\Delta(E, L) \preceq Z$ with the following properties.

(1) $\Delta$ contains $E$ as a component of multiplicity one and the restriction map $H^{0}(Z, L) \rightarrow H^{0}(\Delta, L)$ is surjective.

(2) $\Delta$ is the fundamental cycle on its support and $\mathcal{O}_{\Delta}\left(L-K_{X}\right)$ is numerically trivial.

(3) $E \Delta=\Delta^{2}=-1$ and $\mathcal{O}_{\Delta}\left(L-K_{X}-\Delta\right) \simeq \mathcal{O}_{\Delta}(p)$ for any point $p \in E \backslash$ $\operatorname{Supp}(\Delta-E)$.

(4) $E \subset \mathrm{Bs}\left|K_{\Delta}\right|$ and $\Delta$ decomposes as $\Delta=E+\Gamma_{1}+\cdots+\Gamma_{n-1}$, where $n=-E^{2}$ and the $\Gamma_{i}$ 's are curves with $E \Gamma_{i}=-\Gamma_{i}^{2}=1$ for $i \geq 1$ and $\mathcal{O}_{\Gamma_{i}+\cdots+\Gamma_{n-1}}\left(\Gamma_{i-1}\right) \simeq$ $\mathcal{O}_{\Gamma_{i}+\cdots+\Gamma_{n-1}}$ for $i \geq 2$.

Proof. This is an analogue of Theorem 1.1. We let $\Delta$ be a minimal curve such that $E \preceq \Delta \preceq Z$ and the restriction map $H^{0}(Z, L) \rightarrow H^{0}(\Delta, L)$ is surjective. If $\Delta=E$, then we must have $H^{0}(E, L)=0$, since $E \subset \operatorname{Bs}|L|$. Then the Riemann-Roch theorem shows that $-h^{1}(E, L)=\left.\operatorname{deg} L\right|_{E}+1-p_{a}(E)=(1 / 2)\left(K_{X} E-E^{2}\right)+\left.\operatorname{deg}\left(L-K_{X}\right)\right|_{E}$, which is impossible because $E^{2}<0, K_{X} E \geq 0$ and $\left.\operatorname{deg}\left(L-K_{X}\right)\right|_{E} \geq 0$ by the nefness of $L-K_{X}$ and $K_{X}$. Therefore, $\Delta$ is reducible. Since $K_{\Delta}-L=\Delta-\left(L-K_{X}\right)$ on $\Delta$ and $\Delta^{2}<0$, we see that $K_{\Delta}-L$ is not nef on $\Delta$. Then it follows from [4, Lemma 2.2.1] that $E$ is of multiplicity one in $\Delta, \mathcal{O}_{\Delta-E}\left(\Delta-\left(L-K_{X}\right)\right)$ is nef and the image of $H^{0}(\Delta, L) \rightarrow H^{0}(E, L)$ contains the subspace coming from $H^{0}(E, L-$ $(\Delta-E))$. Since $\mathcal{O}_{\Delta-E}\left(\Delta-\left(L-K_{X}\right)\right)$ and $L-K_{X}$ are nef, we obtain $(\Delta-E) \Delta \geq$ $\left.\operatorname{deg}\left(L-K_{X}\right)\right|_{\Delta-E} \geq 0$. Since $E \subset \mathrm{Bs}|L|$, we must have $h^{0}(E, L-(\Delta-E))=0$. We have $\mathcal{O}_{E}(L-(\Delta-E)) \simeq \mathcal{O}_{E}\left(K_{E}+\left(L-K_{X}\right)-\Delta\right)$ and see that it is non-special, because $E \Delta<0$ and $\left.\operatorname{deg}\left(L-K_{X}\right)\right|_{E} \geq 0$. Then we get $E \simeq \mathbb{P}^{1}, E \Delta=-1$ and $\left.\operatorname{deg}\left(L-K_{X}\right)\right|_{E}=0$ similarly as in the proof of Theorem 1.1. Using $\Delta^{2}<0$, we get $\Delta^{2}=-1$ by $E \Delta=-1$ and $(\Delta-E) \Delta \geq\left.\operatorname{deg}\left(L-K_{X}\right)\right|_{\Delta-E} \geq 0$. Furthermore, we see that $\mathcal{O}_{\Delta}\left(L-K_{X}\right)$ and $\mathcal{O}_{\Delta-E}(\Delta)$ are both numerically trivial. Hence $\Delta$ is the fundamental cycle on its support, because $\Delta$ is 1-connected by Lemma 2.1 and $-\Delta$ is nef on $\operatorname{Supp}(\Delta)$.

We now let $p \in E$ be any non-singular point of $\Delta$. By using $p \in \mathrm{Bs}|L|$ and

$$
0 \rightarrow \mathcal{O}_{\Delta}(L-p) \rightarrow \mathcal{O}_{\Delta}(L) \rightarrow \mathcal{O}_{p} \rightarrow 0,
$$

we get $H^{1}(\Delta, L-p) \neq 0$. Since $\mathcal{O}_{\Delta}(L-p) \simeq \mathcal{O}_{\Delta}\left(K_{\Delta}-\Delta+\left(L-K_{X}\right)-p\right)$ which is numerically equivalent to $K_{\Delta}$, we get $\mathcal{O}_{\Delta}\left(-\Delta+L-K_{X}\right) \simeq \mathcal{O}_{\Delta}(p)$ by the 1 connectedness of $\Delta$. Since $p \in E$ can move, we have $h^{0}\left(\Delta, \mathcal{O}_{\Delta}(p)\right) \geq 2$ which enables 
us to conclude that $p \in \mathrm{Bs}\left|K_{\Delta}\right|$ similarly as in the proof of Theorem 1.1. Therefore, $E \subset \mathrm{Bs}\left|K_{\Delta}\right|$. Then we have the decomposition $\Delta=E+\Gamma_{1}+\cdots+\Gamma_{n-1}$ as in Theorem 1.1. Since $E \Gamma_{i}=1, \Delta \Gamma_{i}=0$ and $\Gamma_{i} \Gamma_{j}=0$ when $j \neq i$, we get $\Gamma_{i}^{2}=-1$. By Lemma $2.1, \Gamma_{i}$ is numerically 1-connected.

By virtue of Lemma 2.1 and the fact $\Delta^{2}=-1$, we see that $\Delta$ is not only a minimal but also the smallest curve with the desired properties.

Definition 2.3. Let $L$ be a line bundle on the fundamental cycle $Z$ with $L-K_{X}$ nef and $E$ an irreducible curve in $\operatorname{Bs}|L|$. We call the curve $\Delta$ as in Proposition 2.2 the loupe for $E$ (with respect to $L$ ).

Now, we are going to show the following theorem which covers the first half of the Main Theorem in Introduction.

TheOREm 2.4. Let $(V, o)$ be a normal surface singular point and $\pi: X \rightarrow V$ the minimal resolution. If $L$ is a line bundle on $X$ such that $L-K_{X}$ is nef on $\pi^{-1}(o)$, then the fixed part of $|L|$ supports at most exceptional sets of rational singular points.

Proof. Let $\mathcal{E}=\bigcup_{i} E_{i}$ be a connected bunch of irreducible curves $E_{i}$ contained in $\operatorname{Bs}|L|$. We sometimes regard $\mathcal{E}$ as a reduced divisor. For each $i$, we take the loupe $\Delta_{i}$ for $E_{i}$ with respect to $L$. Since $\mathcal{E}$ is connected and $\Delta_{i}^{2}=-1$, the set $\left\{\Delta_{i}\right\}$ of loupes has the biggest element $\Delta$ by Lemma 2.1. Then $\mathcal{E} \preceq \Delta$. We let $E \preceq \mathcal{E}$ be the component whose loupe is $\Delta$. Put $E^{2}=-n$. By (4) of Proposition 2.2, $\Delta$ decomposes as $\Delta=E+\Gamma_{1}+\cdots+\Gamma_{n-1}$, where $\Gamma_{\alpha}$ is a 1-connected curve satisfying $E \Gamma_{\alpha}=-\Gamma_{\alpha}^{2}=1$ and $\mathcal{O}_{\Gamma_{\alpha}+\cdots+\Gamma_{n-1}}\left(\Gamma_{\alpha-1}\right) \simeq \mathcal{O}_{\Gamma_{\alpha}+\cdots+\Gamma_{n-1}}$. Recall that the last condition shows either $\operatorname{Supp}\left(\Gamma_{\alpha}\right) \cap \operatorname{Supp}\left(\Gamma_{\beta}\right)=\emptyset$ or $\Gamma_{\beta} \preceq \Gamma_{\alpha}$ when $\alpha<\beta$ (see, Remark 1.2). Note that we cannot have $\Gamma_{\alpha}=\Gamma_{\beta}$ when $\alpha<\beta$, because $\Gamma_{\alpha} \Gamma_{\beta}=0$ and $\Gamma_{\alpha}^{2}=-1$. After changing the labeling if necessary, we may assume that $\left\{E_{1}, \ldots, E_{\ell}\right\}$ is the set of all irreducible components of $\mathcal{E}-E$ with $E E_{i}>0$. For each $i \in\{1, \ldots, \ell\}$, we denote by $\alpha(i)$ the smallest one among those indices $\alpha$ 's with $E_{i} \preceq \Gamma_{\alpha}$. Then every other $\Gamma_{\beta}$ containing $E_{i}$ is a subcurve of $\Gamma_{\alpha(i)}$. Since $E \nprec \Gamma_{\alpha(i)}$ and $E \Gamma_{\alpha(i)}=1$, we get $E E_{i}=1$ and see that $E_{i}$ is the unique component of multiplicity one in $\Gamma_{\alpha(i)}$ which meets $E$. Furthermore, we know that $\Gamma_{\alpha(1)}, \ldots, \Gamma_{\alpha(\ell)}$ are mutually disjoint, because, otherwise, there would be two distinct indices $i, j$ with $\Gamma_{\alpha(i)} \prec \Gamma_{\alpha(j)}$ which would imply $E_{i}=E_{j}$ by what we have just seen. We also remark that, for any $E_{j} \preceq \mathcal{E}, E_{j} \neq E$, we can find a unique $\Gamma_{\alpha(i)}$ such that $E_{j} \preceq \Gamma_{\alpha(i)}$, because $E_{j}$ should be connected to $E$ by a path consisting of curves in $\mathcal{E}$; we would immediately get a contradiction to that $\mathcal{E}$ is connected if there were no such $i$. Put $\mathcal{E}_{i}=(\mathcal{E}-E) \cap \operatorname{Supp}\left(\Gamma_{\alpha(i)}\right)$ for $i=1, \ldots, \ell$. Then $\mathcal{E}_{i}$ is connected and we get the decomposition of $\mathcal{E}-E$ into the connected components: $\mathcal{E}-E=\bigsqcup_{i=1}^{\ell} \mathcal{E}_{i}$.

We denote by $Z_{\mathcal{E}}$ the fundamental cycle on $\mathcal{E}$. Let $G$ be the biggest subcurve of $\Delta$ with $\operatorname{Supp}(G)=\mathcal{E}$. Then $0 \geq E_{j} \Delta=E_{j} G+E_{j}(\Delta-G) \geq E_{j} G$ for any $E_{j} \preceq \mathcal{E}$. This shows that $-G$ is nef on $\mathcal{E}$. It follows $Z_{\mathcal{E}} \preceq G \preceq \Delta$. In particular, we know that $E$ is of multiplicity one in $Z_{\mathcal{E}}$.

Now, we claim that $H^{1}\left(Z_{\mathcal{E}}, \mathcal{O}_{Z_{\mathcal{E}}}\right)=0$. We argue by induction on the number of irreducible components. If $\mathcal{E}$ is a single curve, then we clearly have $Z_{\mathcal{E}}=\mathcal{E} \simeq \mathbb{P}^{1}$ and the assertion follows. We assume that $\mathcal{E}$ consists of several irreducible components. Let $\mathcal{E}_{i}$ be as above. Since the number of irreducible components of $\mathcal{E}_{i}$ is strictly smaller than that of $\mathcal{E}$, by the hypothesis of the induction, we have $H^{1}\left(Z_{\mathcal{E}_{i}}, \mathcal{O}_{Z_{\mathcal{E}_{i}}}\right)=0$ for the fundamental cycle $Z_{\mathcal{E}_{i}}$ on $\mathcal{E}_{i}$. This implies that we obtain a rational singular point by contracting $\mathcal{E}_{i}$. Hence, if $G_{i}$ denotes the biggest subcurve of $Z_{\mathcal{E}}$ supported on $\mathcal{E}_{i}$, 
then we also have $H^{1}\left(G_{i}, \mathcal{O}_{G_{i}}\right)=0$ (see [1], [2]). Recall that $E$ is of multiplicity one in $Z_{\mathcal{E}}$. Since $Z_{\mathcal{E}}-E=G_{1}+\cdots+G_{\ell}$ and $\operatorname{Supp}\left(G_{i}\right) \cap \operatorname{Supp}\left(G_{j}\right)=\emptyset$ when $i \neq j$, we get $H^{1}\left(Z_{\mathcal{E}}-E, \mathcal{O}_{Z_{\mathcal{E}} E}\right)=\bigoplus_{i=1}^{\ell} H^{1}\left(G_{i}, \mathcal{O}_{G_{i}}\right)=0$. Let $\eta$ be a section of the line bundle $\left[\Delta-Z_{\mathcal{E}}\right]$ defining the curve $\Delta-Z_{\mathcal{E}}$. Since $E$ is not a component of $\Delta-Z_{\mathcal{E}}$, $\eta$ induces an effective divisor on $E$. The restriction maps and the natural injections induced by $\eta$ give us the following commutative diagram.

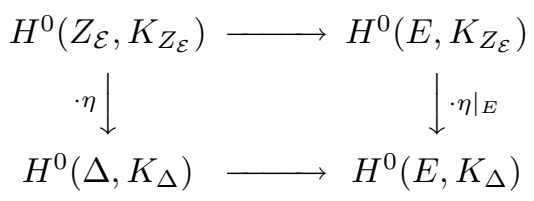

Since $E \subset \operatorname{Bs}\left|K_{\Delta}\right|$, the map at the bottom row is the zero map. Hence $H^{0}\left(Z_{\mathcal{E}}, K_{Z_{\mathcal{E}}}\right) \rightarrow$ $H^{0}\left(E, K_{Z_{\mathcal{E}}}\right)$ is also the zero map. Note that its kernel is isomorphic to $H^{0}\left(Z_{\mathcal{E}}-\right.$ $\left.E, K_{Z_{\mathcal{E}}-E}\right)$ whose dual is $H^{1}\left(Z_{\mathcal{E}}-E, \mathcal{O}_{Z_{\mathcal{E}}-E}\right)$ which is zero as we saw above. It follows that $H^{0}\left(Z_{\mathcal{E}}, K_{Z_{\mathcal{E}}}\right)=0$. Then we get $H^{1}\left(Z_{\mathcal{E}}, \mathcal{O}_{Z_{\mathcal{E}}}\right)=0$ as desired, by the Serre duality theorem.

This shows that $Z_{\mathcal{E}}$ is the fundamental cycle of a rational singular point and completes the proof of Theorem 2.4.

REMARK 2.5. (1) We do not know whether the restriction map $H^{0}(Z, L) \rightarrow$ $H^{0}\left(Z_{\mathcal{E}}, L\right)$ is the zero map or not. This explains a reason why we need a round-about argument as above.

(2) $\Delta$ and $Z_{\mathcal{E}}$ are the fundamental cycles on their respective supports. Since $\Delta^{2}=-1$ and $K_{X}$ is nef, we have $p_{a}(\Delta)>0$ while we know $p_{a}\left(Z_{\mathcal{E}}\right)=0$. It follows that we have not only $Z_{\mathcal{E}} \preceq \Delta$ but also that $\mathcal{E}$ is strictly smaller than $\operatorname{Supp}(\Delta)$. This also shows that if $(V, o)$ is rational, then $|L|$ has no fixed components.

(3) By Proposition $1.5, Z_{\mathcal{E}}$ decomposes as

$$
Z_{\mathcal{E}}=E+Z_{1}+\cdots+Z_{k}
$$

where $k=E\left(Z_{\mathcal{E}}-E\right)$, each $Z_{i}$ is the fundamental cycle on its support with $E Z_{i}=1$ and $\mathcal{O}_{Z_{j}+\cdots+Z_{k}}\left(-Z_{j-1}\right) \simeq \mathcal{O}_{Z_{j}+\cdots+Z_{k}}$ for $2 \leq j \leq k$. This may be useful to study the configuration of $\mathcal{E}$. We also remark that $E Z_{\mathcal{E}} \leq E Z_{\mathcal{E}}+E\left(\Delta-Z_{\mathcal{E}}\right)=E \Delta=-1$.

There are three basic invariants of $(V, o)$ (cf. [11]): The geometric genus $p_{g}(V, o)=$ $\operatorname{dim}\left(R^{1} \pi_{*} \mathcal{O}_{X}\right)_{o}$, the arithmetic genus $p_{a}(V, o)=\sup \left\{p_{a}(D) \mid 0 \prec D, \operatorname{Supp}(D) \subset\right.$ $\left.\pi^{-1}(o)\right\}$ and the fundamental genus $p_{f}(V, o)=p_{a}(Z)$. We have $p_{f}(V, o) \leq p_{a}(V, o) \leq$ $p_{g}(V, o)$. Note that the inequalities are strict in many cases. For example, if $(V, o)$ is a hypersurface singularity defined by $x^{3}+y^{4}+z^{12}=0$, then $p_{f}=3, p_{a}=4$ and $p_{g}=8$.

The following completes the proof of the Main Theorem.

Proposition 2.6. Let $(V, o)$ be a normal surface singularity and $\pi: X \rightarrow V$ the minimal resolution. Let $L$ be a line bundle on $X$ such that $L-K_{X}$ is nef and assume that $|L|$ has a fixed component.

(1) If $(U, p)$ denotes the rational singular point obtained by contracting a connected component of the fixed part of $|L|$, then the multiplicity mult $(U, p)$ and the embedding dimension $\operatorname{embdim}(U, p)$ satisfy

$$
\operatorname{mult}(U, p) \leq 2 p_{f}(V, o), \quad \operatorname{embdim}(U, p) \leq 2 p_{f}(V, o)+1 .
$$


(2) If an irreducible curve $E$ is a fixed component of $|L|$, then $-E^{2} \leq p_{f}(V, o)+1$.

Proof. (1) We retain the notation and assumptions as in the proof of Theorem 2.4. $\mathcal{E}$ is now the connected component of $\mathrm{Bs}|L|$ which produces $(U, p)$. Since $Z_{\mathcal{E}}$ is the fundamental cycle of a rational singularity, it is numerically 1-connected and we have $-Z_{\mathcal{E}}^{2}=K_{X} Z_{\mathcal{E}}+2$ by the adjunction formula. Since $K_{X}$ is nef and $Z_{\mathcal{E}} \prec \Delta$, we have $K_{X} Z_{\mathcal{E}} \leq K_{X} \Delta=2 p_{a}(\Delta)-1$ by $\Delta^{2}=-1$. Assume that $K_{X} Z_{\mathcal{E}}=K_{X} \Delta$. Then $\Delta-Z_{\mathcal{E}}$ consists of $(-2)$-curves. Since $\Delta$ is numerically 1 -connected, $\operatorname{Supp}(\Delta)$ is a connected set. Furthermore, we know that $\mathcal{E}$ is a proper subset of $\operatorname{Supp}(\Delta)$ (see, Remark 2.5, (2)). It follows that there exists an irreducible curve $A$ contained in the closure of $\operatorname{Supp}(\Delta) \backslash \mathcal{E}$ that meets $\mathcal{E}$. On the other hand, since $A$ is a $(-2)$-curve and $\mathcal{O}_{A}\left(K_{X}\right) \simeq \mathcal{O}_{A}(L)$ by (2) of Proposition 2.2, any section of $L$ is constant on $A$, which should be zero because $A$ meets $\mathcal{E}$. Hence $A \subset \mathrm{Bs}|L|$. This contradicts that $\mathcal{E}$ is a connected component of $\mathrm{Bs}|L|$. Therefore, $K_{X} Z_{\mathcal{E}}$ is strictly smaller than $K_{X} \Delta$. Then we get $K_{X} Z_{\mathcal{E}} \leq 2 p_{a}(\Delta)-2$ which implies $-Z_{\mathcal{E}}^{2} \leq 2 p_{a}(\Delta)$. Since $\Delta \preceq Z$, we have $p_{a}(\Delta) \leq p_{a}(Z)=p_{f}(V, o)$. In sum, we have shown $-Z_{\mathcal{E}}^{2} \leq 2 p_{f}(V, o)$. Now, the assertion follows from M. Artin's formulas: $\operatorname{mult}(U, p)=-Z_{\mathcal{E}}^{2}$ and $\operatorname{embdim}(U, p)=$ $-Z_{\mathcal{E}}^{2}+1$.

(2) We already know that $E \simeq \mathbb{P}^{1}$. Let $\Delta$ be the loupe for $E$ with respect to $L$. By Proposition 2.2, it is a numerically 1-connected curve with $\Delta^{2}=E \Delta=-1$ and $E \subset \mathrm{Bs}\left|K_{\Delta}\right|$. Furthermore, we know that $\mathcal{O}_{\Delta-E}(\Delta)$ is numerically trivial. Then $\left.\operatorname{deg} K_{\Delta}\right|_{A}=\left.\operatorname{deg} K_{X}\right|_{A}+A \Delta=\left.\operatorname{deg} K_{X}\right|_{A} \geq 0$ for any irreducible component $A$ of $\Delta-E$, that is, $K_{\Delta}$ is nef on $\Delta-E$. Recall that $E$ is of multiplicity one in $\Delta$. Then we can show that $(\Delta-E) E \leq p_{a}(\Delta)$ holds similarly as in the proof of Corollary 1.3. Therefore, $-E^{2} \leq p_{a}(\Delta)+1 \leq p_{f}(V, o)+1$.

The bound in (2) is sharp, while (1) may be rather weak when $p_{f}(V, o)>1$.

When $(V, o)$ is a rational singular point, Proposition 2.6 implies that $|L|$ is free from fixed components whenever $L-K_{X}$ is nef. Since $K_{X}$ is nef and $H^{1}\left(Z, \mathcal{O}_{Z}\right)=0$, the following slightly more general result shows that, in fact, we have $\operatorname{Bs}|L|=\emptyset$ in this case.

Proposition 2.7. Let $D$ be an effective non-zero divisor with $H^{1}\left(D, \mathcal{O}_{D}\right)=0$ on a smooth surface. If $M$ is a nef line bundle on $D$, then the restriction map $H^{0}(D, M) \rightarrow H^{0}(A, M)$ is surjective for any irreducible component $A$ of $D$. In particular, $\mathrm{Bs}|M|=\emptyset$ when $M$ is nef.

Proof. First, we notice that $H^{1}\left(D^{\prime}, \mathcal{O}_{D^{\prime}}\right)=0$ holds for any effective divisor $D^{\prime}$ with $D^{\prime} \preceq D$, since $H^{1}\left(D, \mathcal{O}_{D}\right)=0$. In particular, we have $A \simeq \mathbb{P}^{1}$. Let $\Delta$ be a minimal effective divisor with $A \preceq \Delta \preceq D$ such that the restriction map $H^{0}(D, M) \rightarrow$ $H^{0}(\Delta, M)$ is surjective. Since $H^{1}\left(\Delta, \mathcal{O}_{\Delta}\right)=0$, we have $p_{a}(\Delta) \leq 0$ and, hence, $\operatorname{deg} K_{\Delta}=2 p_{a}(\Delta)-2 \leq-2$. It follows that $K_{\Delta}-M$ is not nef on $\Delta$. By [4, Lemma 2.2.1], $A$ is of multiplicity one in $\Delta$ and $K_{\Delta}-M$ is nef on $\Delta-A$. Then

$$
\operatorname{deg} K_{\Delta}=\left.\operatorname{deg} K_{\Delta}\right|_{\Delta-A}+\left.\operatorname{deg} K_{\Delta}\right|_{A} \geq\left.\operatorname{deg} M\right|_{\Delta-A}+\operatorname{deg} K_{A}+(\Delta-A) A
$$

Notice that we have $\operatorname{deg} K_{\Delta} \leq-2, \operatorname{deg} K_{A}=-2$ and $\left.\operatorname{deg} M\right|_{\Delta-A} \geq 0$. Then $(\Delta-$ $A) A \leq 0$. On the other hand, since $A$ is of multiplicity one in $\Delta$, we have $(\Delta-A) A \geq 0$. Hence $(\Delta-A) A=0$ and it follows $A \cap(\Delta-A)=\emptyset$. Then $H^{0}(\Delta, M)=H^{0}(A, M) \oplus$ $H^{0}(\Delta-A, M)$ and the restriction $H^{0}(\Delta, M) \rightarrow H^{0}(A, M)$ is surjective. Hence we must have $\Delta=A$ by the minimality of $\Delta$. $\square$ 
3. Further remarks. Here, we state some properties of loupes not needed for the proof of Theorem 2.4 for the later use.

Let $L$ be as before a line bundle with $L-K_{X}$ nef. Take an irreducible curve $E \subset \mathrm{Bs}|L|$ and let $\Delta$ be the loupe for $E$ with respect to $L$. Put $E^{2}=-n$ and let

$$
\Delta=E+\Gamma_{1}+\cdots+\Gamma_{n-1}
$$

be the decomposition of $\Delta$ as in (4) of Proposition 2.2. Then we have $\mathcal{O}_{\Gamma_{i}+\cdots+\Gamma_{n-1}}\left(\Gamma_{i-1}\right) \simeq \mathcal{O}_{\Gamma_{i}+\cdots+\Gamma_{n-1}}$ for $i \geq 2$ which implies either $\Gamma_{j} \prec \Gamma_{i}$ or $\operatorname{Supp}\left(\Gamma_{i}\right) \cap \operatorname{Supp}\left(\Gamma_{j}\right)=\emptyset$ when $i<j$. Since $E$ is of multiplicity one in $\Delta$ and $E \Gamma_{i}=1$, each $\Gamma_{i}$ has a unique irreducible component of multiplicity one which intersects $E$ at a point.

LEMma 3.1. Each $\Gamma_{i}$ is the fundamental cycle on its support and $-\Gamma_{i}-\sum_{\Gamma_{j} \prec \Gamma_{i}} \Gamma_{j}$ is nef on $\Gamma_{i}$. There exists a unique irreducible component $A_{i} \preceq \Gamma_{i}$ of multiplicity one such that $A_{i} \Gamma_{i}=-1$ and $C \Gamma_{i}=0$ for any curve with $C \preceq \Gamma_{i}-A_{i}$. Furthermore, $A_{i} \neq A_{j}$ for $j \neq i$, and $A_{i} E=1$ holds if and only if $\Gamma_{i}$ is minimal in $\left\{\Gamma_{\nu}\right\}_{\nu=1}^{n-1}$.

Proof. The first assertion follows from Lemma 1.4. Since $\Gamma_{i}^{2}=-1$ and $\mathcal{O}_{\Gamma_{i}}\left(-\Gamma_{i}\right)$ is nef, one immediately sees that $A_{i}$ as in the statement exists. When $i<j, \mathcal{O}_{\Gamma_{j}}\left(\Gamma_{i}\right)$ is numerically trivial by Proposition 2.2, (4), and we get $A_{j} \Gamma_{i}=0$. Similarly, we get $A_{i} \Gamma_{j}=0$ when $j<i$. Hence $A_{j} \neq A_{i}$ for $j \neq i$. Let $k$ be the biggest index such that $\Gamma_{k} \preceq \Gamma_{i}$. Since $\mathcal{O}_{\Delta-E}(\Delta)$ is numerically trivial by Proposition 2.2, we obtain $A_{k} E=1$ from $0=A_{k} \Delta=A_{k} E+A_{k} \Gamma_{k}$. It follows that $A_{k}$ is the unique component of $\Gamma_{i}$ which meets $E$.

The following will be useful when we study the configuration of the fixed part by an inductive argument.

Proposition 3.2. Assume that $\Gamma_{i}$ is minimal in $\left\{\Gamma_{1}, \ldots, \Gamma_{n-1}\right\}$ and let $A_{i} \preceq \Gamma_{i}$ be the curve as in the previous lemma. Then $\mathcal{O}_{\Gamma_{i}}\left(L-K_{X}-\Gamma_{i}\right) \simeq \mathcal{O}_{\Gamma_{i}}\left(p_{i}\right)$, where $p_{i}=A_{i} \cap E$. Furthermore, $\Gamma_{i}$ is a minimal curve among those curves $C$ with $A_{i} \preceq$ $C \preceq Z$ such that $H^{0}(X, L) \rightarrow H^{0}(C, L)$ is surjective. In particular, $\Gamma_{i}$ is the loupe for $A_{i}$ with respect to $L$, when $A_{i} \subset \mathrm{Bs}|L|$.

Proof. Note that we have $\mathcal{O}_{\Gamma_{i}}\left(\Gamma_{j}\right) \simeq \mathcal{O}_{\Gamma_{i}}$ for any $j \neq i$ by the choice of $\Gamma_{i}$. It follows $\mathcal{O}_{\Gamma_{i}} \simeq \mathcal{O}_{\Gamma_{i}}\left(K_{X}-L+\Delta\right) \simeq \mathcal{O}_{\Gamma_{i}}\left(K_{X}-L+\Gamma_{i}+E\right)$. Hence we get $\mathcal{O}_{\Gamma_{i}}(L-$ $\left.K_{X}-\Gamma_{i}\right) \simeq \mathcal{O}_{\Gamma_{i}}\left(p_{i}\right)$.

Before showing the second assertion, we notice that the restriction map $H^{0}(\Delta, L) \rightarrow H^{0}\left(\Gamma_{\alpha}, L\right)$ is surjective for any $\alpha \in\{1, \ldots, n-1\}$. To see this, consider the cohomology long exact sequence for

$$
0 \rightarrow \mathcal{O}_{\Delta-\Gamma_{\alpha}}\left(L-\Gamma_{\alpha}\right) \rightarrow \mathcal{O}_{\Delta}(L) \rightarrow \mathcal{O}_{\Gamma_{\alpha}}(L) \rightarrow 0 .
$$

We know that $\Delta-\Gamma_{\alpha}$ is numerically 1-connected, because $\left(\Delta-\Gamma_{\alpha}\right) \Gamma_{\alpha}=1$. We have $\mathcal{O}_{\Delta-\Gamma_{\alpha}}\left(L-\Gamma_{\alpha}\right) \simeq \mathcal{O}_{\Delta-\Gamma_{\alpha}}\left(K_{\Delta-\Gamma_{\alpha}}+L-K_{X}-\Delta\right)$. Since $\mathcal{O}_{\Delta-\Gamma_{\alpha}}\left(L-K_{X}-\Delta\right)$ is nef of degree one, we get $H^{1}\left(\Delta-\Gamma_{\alpha}, L-\Gamma_{\alpha}\right)=0$. Hence $H^{0}(\Delta, L) \rightarrow H^{0}\left(\Gamma_{\alpha}, L\right)$ is surjective.

Let $C$ be any proper subcurve of $\Gamma_{i}$ with $A_{i} \preceq C$. Then $\mathcal{O}_{\Gamma_{i}-C}\left(L-K_{X}-\Gamma_{i}\right) \simeq$ $\mathcal{O}_{\Gamma_{i}-C}$ by $\mathcal{O}_{\Gamma_{i}}\left(L-K_{X}-\Gamma_{i}\right) \simeq \mathcal{O}_{\Gamma_{i}}\left(p_{i}\right)$. It follows that $\mathcal{O}_{\Gamma_{i}-C}(L-C) \simeq \omega_{\Gamma_{i}-C}$. Consider the cohomology long exact sequence for

$$
0 \rightarrow \mathcal{O}_{\Gamma_{i}-C}(L-C) \rightarrow \mathcal{O}_{\Gamma_{i}}(L) \rightarrow \mathcal{O}_{C}(L) \rightarrow 0 .
$$


We have $h^{1}\left(\Gamma_{i}-C, L-C\right)=h^{1}\left(\Gamma_{i}-C, \omega_{\Gamma_{i}-C}\right) \neq 0$ and $H^{1}\left(\Gamma_{i}, L\right)=0$. Hence the restriction map $H^{0}\left(\Gamma_{i}, L\right) \rightarrow H^{0}(C, L)$ cannot be surjective. This means that $\Gamma_{i}$ is a minimal curve with $A_{i} \preceq \Gamma_{i} \preceq Z$ such that $H^{0}(X, L) \rightarrow H^{0}\left(\Gamma_{i}, L\right)$ is surjective.

Though the following can be shown by using the A-D-E classification and the fact given in Remark 2.5 that there is a non-multiple component $E \preceq Z_{\mathcal{E}}$ with $E Z_{\mathcal{E}}<0$, we present the proof as an application of Proposition 3.2 for the use in the next section.

Corollary 3.3. Let $(V, o)$ be a normal surface singularity and $\pi: X \rightarrow V$ the minimal resolution. Take a line bundle $L$ on $X$ such that $L-K_{X}$ is nef. Let $\mathcal{E}=\bigcup_{i=0}^{m-1} E_{i}$ be a connected bunch of irreducible curves $E_{i} \subset \mathrm{Bs}|L|$. If the singular point obtained by contracting $\mathcal{E}$ is a rational double point, then it is of type $\mathbf{A}_{m}$.

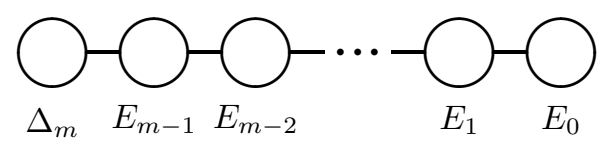

FIG. 1.

Proof. We know that all the $E_{i}$ 's are (-2)-curves. Take the loupe $\Delta_{i}$ for $E_{i}$ with respect to $L$. Since $E_{i}^{2}=-2$, the decomposition of $\Delta_{i}$ as in Proposition 2.2 is of the form $E_{i}+\Gamma_{i, 1}$. By Proposition 3.2, we can assume that $\Gamma_{i, 1}=\Delta_{i+1}$ for $i \in$ $\{0, \ldots, m-2\}$ after changing the ordering of the $E_{j}$ 's if necessary. Put $\Delta_{m}:=\Gamma_{m-1,1}$. Then $\Delta_{m} \prec \cdots \prec \Delta_{1} \prec \Delta_{0}$ and $E_{i}=\Delta_{i}-\Delta_{i+1}$ for $i \in\{0, \ldots, m-1\}$. Furthermore, we have $E_{i} E_{i+1}=1$ for $i \in\{0, \ldots, m-2\}$ and $E_{i} E_{j}=0$ when $|i-j|>1$. Hence $\mathcal{E}$ corresponds to the rational double point of type $\mathbf{A}_{m}$, and (a part of) the dual graph of $\Delta_{0}$ is as in Fig. 1.

In particular, this suggests that we cannot produce an arbitrary rational singular point by contracting the canonical fixed part.

Finally in this section, we want to emphasize the importance of the study of $\left|K_{\Delta}\right|$ by showing that $\left|K_{\Delta}\right|$ faithfully inherits information on the base locus of $|L|$.

Lemma 3.4. For any subcurve $C \prec \Delta$, the restriction map $H^{0}(X, L) \rightarrow H^{0}(C, L)$ is the zero map if and only if so is the map $H^{0}\left(\Delta, K_{\Delta}\right) \rightarrow H^{0}\left(C, K_{\Delta}\right)$. Furthermore, $\mathrm{Bs}\left|K_{\Delta}\right|=\operatorname{Bs}|L| \cap \operatorname{Supp}(\Delta)$.

Proof. Recall that the restriction map $H^{0}(X, L) \rightarrow H^{0}(\Delta, L)$ is surjective and $\mathcal{O}_{\Delta}\left(K_{\Delta}\right) \simeq \mathcal{O}_{\Delta}(L-p)$ for a general point $p \in E$ by Proposition 2.2. Consider the commutative diagram

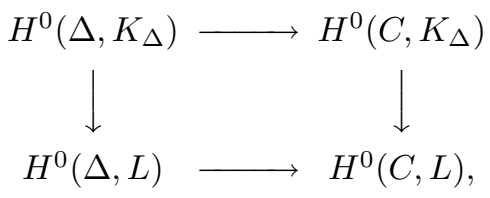

where the horizontal maps are restrictions and the vertical maps are natural inclusions induced by $\mathcal{O}_{\Delta}(L-p) \hookrightarrow \mathcal{O}_{\Delta}(L)$. Since $p \in E \subset \mathrm{Bs}|L|, H^{0}\left(\Delta, K_{\Delta}\right) \rightarrow H^{0}(\Delta, L)$ is an isomorphism. Hence the first assertion follows from the above diagram. As to the second assertion, we only have to note that an isolated base point, if exists, is on $\Delta-E$. $\mathrm{Q}$ 
4. Weakly elliptic singularities. A normal surface singularity $(V, o)$ is called a numerically Gorenstein singularity if there exists a curve $Z_{K}$ such that $-Z_{K}$ is numerically equivalent to $K_{X}$ on $\pi^{-1}(o)$. Such a curve $Z_{K}$ is called the canonical cycle and the geometric genus of $(V, o)$ is given by $p_{g}(V, o)=h^{1}\left(Z_{K}, \mathcal{O}_{Z_{K}}\right)$ (see e.g., [10]). A normal surface singularity $(V, o)$ is called an elliptic singularity [11] if $p_{a}(V, o)=$ 1. If an elliptic singularity is numerically Gorenstein, then $Z_{K}$ is a numerically 0connected curve, and S.S.T. Yau [13] introduced a sequence $\left\{Z_{i}\right\}_{i=0}^{n}$ of curves with $Z_{K}=\sum_{i=0}^{n} Z_{i}$, called the elliptic sequence, where the $Z_{i}$ 's are the fundamental cycles on their respective supports (since we are on the minimal resolution), $Z_{n} \prec Z_{n-1} \prec$ $\ldots \prec Z_{1} \prec Z_{0}=Z$ and $Z_{n}$ is contracted to an elliptic Gorenstein singularity with $p_{g}=1$ (a minimally elliptic singularity). It is shown in [13] that $p_{g}(V, o)$ is at most $n+1$, that is, the length of the elliptic sequence.

TheOREm 4.1. Let $(V, o)$ be a normal surface singularity and $\pi: X \rightarrow V$ the minimal resolution. Let $L$ be a line bundle on $X$ such that $L-K_{X}$ is nef, and suppose that $|L|$ has a fixed component. Then the following hold.

(1) If $(V, o)$ is an elliptic singularity, then the fixed part of $|L|$ supports at most exceptional sets of rational double points of type $\mathbf{A}$.

(2) Let $\bigcup_{i=0}^{m-1} E_{i}$ be a connected component of the fixed part of $|L|$ which supports the exceptional set of the rational double point of type $\mathbf{A}_{m}$. Take the loupe $\Delta_{i}$ for $E_{i}$ with respect to $L$, and change the indices so that $\Delta_{m-1} \prec \cdots \prec \Delta_{1} \prec \Delta_{0}$ if necessary. Suppose that $p_{a}\left(\Delta_{0}\right)=1$. Then $\Delta_{0}$ is contracted to an elliptic numerically Gorenstein singularity and $\left\{\Delta_{i}\right\}_{i=0}^{m}$ forms its elliptic sequence, where $\Delta_{m}=\Delta_{0}-\sum_{i=0}^{m-1} E_{i}$. Furthermore, $\mathcal{O}_{\Delta_{m}}\left(L-K_{X}\right) \simeq \mathcal{O}_{\Delta_{m}}\left(\Delta_{m-1}\right)$.

Proof. The assertion (1) follows from Corollary 3.3, because $(U, p)$ as in Proposition 2.6 should be a rational double point if $p_{a}(V, o)=1$. In order to show (2), we employ the same notation and conventions as in the proof of Corollary 3.3, assuming now that $\mathcal{E}$ is a connected component of the fixed part. Recall that $\mathcal{O}_{\Delta_{0}}\left(L-K_{X}\right)$ is numerically trivial by Proposition 2.2, (2). By Proposition 3.2, $\Delta_{m}=\Delta_{0}-\sum_{i=0}^{m-1} E_{i}$ inherits nice properties from the preceding $\Delta_{i}$ 's: We have $\Delta_{m}^{2}=-1$ and there exists a unique irreducible component $E_{m}$ of multiplicity one in $\Delta_{m}$ with $E_{m-1} E_{m}=1$, $E_{m} \Delta_{m}=-1$ and $\mathcal{O}_{\Delta_{m}}\left(L-K_{X}-\Delta_{m}\right) \simeq \mathcal{O}_{\Delta_{m}}(p)$, where $p=E_{m-1} \cap E_{m}$. We also know that $\Delta_{m}$ is the fundamental cycle on its support.

Suppose that $p_{a}\left(\Delta_{0}\right)=1$. By [11] and [5], we obtain an elliptic singularity by contracting $\Delta_{0}$, since $p_{a}\left(\Delta_{0}\right)=1$ and $\Delta_{0}$ is the fundamental cycle. We know that $\Delta_{i}$ is the fundamental cycle on its support and $\mathcal{O}_{\Delta_{i}-E_{i}}\left(L-K_{X}-\Delta_{i}\right) \simeq \mathcal{O}_{\Delta_{i}-E_{i}}$ for any $i \in\{0, \ldots, m\}$. Since $\Delta_{i}^{2}=-1$ and $K_{X}$ is nef, we get $p_{a}\left(\Delta_{i}\right) \geq 1$ by $0 \leq K_{X} \Delta_{i}=2 p_{a}\left(\Delta_{i}\right)-1$. On the other hand, since $\Delta_{i}$ is a 1-connected curve, we have $p_{a}\left(\Delta_{i}\right)=h^{1}\left(\Delta_{i}, \mathcal{O}_{\Delta_{i}}\right) \leq h^{1}\left(\Delta_{0}, \mathcal{O}_{\Delta_{0}}\right)=1$. Hence $p_{a}\left(\Delta_{i}\right)=1$. Since $\Delta_{i}=\Delta_{1}-E_{1}-\cdots-E_{i-1}$ and $\Delta_{i}$ does not meet $E_{0}+\cdots+E_{i-2}$, we have the following:

CLAIM 4.2. $\mathcal{O}_{\Delta_{i}}\left(L-K_{X}-\Delta_{j}\right) \simeq \mathcal{O}_{\Delta_{i}}$ when $i>j$ and $\mathcal{O}_{\Delta_{i}}\left(L-K_{X}-\Delta_{i}\right) \simeq$ $\mathcal{O}_{\Delta_{i}}\left(E_{i-1}\right)$ when $i>0$.

We remark that $E_{m}$ is not a (-2)-curve, because, otherwise, we have $\left.\operatorname{deg} L\right|_{E_{m}}=$ $\left.\operatorname{deg} K_{X}\right|_{E_{m}}=0$ and the property $E_{m-1} E_{m}=1$ would imply that $E_{m}$ has to be a fixed component of $|L|$, contradicting that $\mathcal{E}$ is a connected component. Then $E_{m}$ is the unique irreducible component of $\Delta_{0}$ which has positive intersection with $K_{X}$. In fact, we have $K_{X} E_{m}=1$ by $K_{X} \Delta_{0}=1$. 
Claim 4.3. $\Delta_{m}$ is numerically 2-connected.

Proof. Let $\Delta_{m}=A+B$ be any effective decomposition. We may assume that $E_{m} \preceq A$. Then $B \Delta_{m}=0$. Furthermore, we have $K_{X} B=0$ which implies that $B^{2}$ is a negative even integer. Then $0=\Delta_{m} B=A B+B^{2} \leq A B-2$. Hence $\Delta_{m}$ is 2-connected.

Since $\Delta_{m}$ is a 2 -connected curve with $p_{a}\left(\Delta_{m}\right)=1$, we have $\omega_{\Delta_{m}} \simeq \mathcal{O}_{\Delta_{m}}$ by $[3$, Proposition (A.7)]. This implies that $\Delta_{m}$ is the fundamental cycle of an elliptic Gorenstein singularity with $p_{g}=1$ (a minimally elliptic singularity). Now, put $\tilde{\Delta}_{i}=$ $\Delta_{i}+\Delta_{i+1}+\cdots+\Delta_{m}$ for $i \in\{0, \ldots, m\}$.

Claim 4.4. $-\tilde{\Delta}_{i}$ is numerically equivalent to $K_{X}$ on $\Delta_{i}$ for any $i \in\{0, \ldots, m\}$. Furthermore, $\mathcal{O}_{\Delta_{i}}\left(K_{X}\right) \simeq \mathcal{O}_{\Delta_{i}}\left(-\tilde{\Delta}_{i}\right)$ holds if and only if $\mathcal{O}_{\Delta_{m}}\left((m-i)\left(L-K_{X}\right)\right) \simeq$ $\mathcal{O}_{\Delta_{m}}$.

Proof. We already know that $\omega_{\Delta_{m}} \simeq \mathcal{O}_{\Delta_{m}}$, that is, $\mathcal{O}_{\Delta_{m}}\left(K_{X}\right) \simeq \mathcal{O}_{\Delta_{m}}\left(-\Delta_{m}\right)$. This gives us the assertion for $i=m$.

We may assume that $i<m$. The fact that $-\tilde{\Delta}_{i}$ is numerically equivalent to $K_{X}$ on $\Delta_{i}$ easily follows from $K_{X} \Delta_{i}=1$ and Claim 4.2 , if we note that $L-K_{X}$ is numerically equivalent to zero on $\Delta_{0}$.

Since $\Delta_{i}$ is numerically 1 -connected, $\mathcal{O}_{\Delta_{i}}\left(K_{X}\right) \simeq \mathcal{O}_{\Delta_{i}}\left(-\tilde{\Delta}_{i}\right)$ is equivalent to $H^{0}\left(\Delta_{i}, K_{X}+\tilde{\Delta}_{i}\right) \neq 0$. We consider the exact sequence

$$
0 \rightarrow \mathcal{O}_{E_{i}+\cdots+E_{m-1}}\left(K_{X}+\tilde{\Delta}_{i}-\Delta_{m}\right) \rightarrow \mathcal{O}_{\Delta_{i}}\left(K_{X}+\tilde{\Delta}_{i}\right) \rightarrow \mathcal{O}_{\Delta_{m}}\left(K_{X}+\tilde{\Delta}_{i}\right) \rightarrow 0
$$

Since $K_{X}+\tilde{\Delta}_{i}-\Delta_{m}$ is anti-nef of degree -1 on the numerically 1-connected rational curve $E_{i}+\cdots+E_{m-1}$, we get $H^{q}\left(E_{i}+\cdots+E_{m-1}, K_{X}+\tilde{\Delta}_{i}-\Delta_{m}\right)=0$ for $q=0,1$. Hence $H^{0}\left(\Delta_{i}, K_{X}+\tilde{\Delta}_{i}\right) \simeq H^{0}\left(\Delta_{m}, \mathcal{O}_{\Delta_{m}}\left(K_{X}+\tilde{\Delta}_{i}\right)\right)$. It follows from Claim 4.2 that

$$
\mathcal{O}_{\Delta_{m}}\left(K_{X}+\tilde{\Delta}_{i}\right) \simeq \mathcal{O}_{\Delta_{m}}\left((m-i)\left(L-K_{X}\right)+K_{X}+\Delta_{m}\right) \simeq \mathcal{O}_{\Delta_{m}}\left((m-i)\left(L-K_{X}\right)\right) .
$$

Therefore, we have $\mathcal{O}_{\Delta_{i}}\left(K_{X}\right) \simeq \mathcal{O}_{\Delta_{i}}\left(-\tilde{\Delta}_{i}\right)$ if and only if $\mathcal{O}_{\Delta_{m}}\left((m-i)\left(L-K_{X}\right)\right) \simeq$ $\mathcal{O}_{\Delta_{m}} \cdot \square$

We have shown that $\tilde{\Delta}_{i}$ is the canonical cycle for the elliptic numerically Gorenstein singularity $\left(V_{i}, p_{i}\right)$ obtained by contracting $\Delta_{i}$ and that $\left\{\Delta_{j}\right\}_{j=i}^{m}$ is the elliptic sequence for each $i$ with $0 \leq i \leq m$. We have $\mathcal{O}_{\Delta_{m}}(L) \simeq \mathcal{O}_{\Delta_{m}}\left(K_{X}+\Delta_{m-1}\right)$ by Claim 4.2. Therefore, we get (2) of Theorem 4.1.

The converse of Theorem 4.1, (2) holds in the following sense.

Proposition 4.5. Let $(V, o)$ be an elliptic numerically Gorenstein singularity with $p_{g}(V, o) \geq 2$ whose fundamental cycle satisfies $Z^{2}=-1$ on the minimal resolution $X$. Let $\left\{Z_{i}\right\}_{i=0}^{n}, Z_{0}=Z$, be the elliptic sequence. If $L$ is a line bundle on $X$ numerically equivalent to $K_{X}$, then either $\mathrm{Bs}|L|$ is one point (which is a non-singular point of $Z_{n}$ ) or it supports the exceptional set of the rational double point of type $\mathbf{A}_{n}$. The latter happens if and only if $\mathcal{O}_{Z_{n}}\left(L-K_{X}\right) \simeq \mathcal{O}_{Z_{n}}\left(Z_{n-1}\right)$.

Proof. Since $Z^{2}=-1$, the minimal resolution dual graph is classified (see e.g., [12] and [9, Proposition 5.13]) which is much similar to one in Fig. 1 . We see that $C_{i}=Z_{i}-Z_{i+1}$ is a $(-2)$-curve for $0 \leq i \leq n-1$ and that $Z_{n}$ is the fundamental cycle of an elliptic Gorenstein singularity with $p_{g}=1$. Furthermore, the dual graph of $C_{0}+\cdots+C_{n-1}$ is of Dynkin type $\mathbf{A}_{n}$ with $C_{j-1} C_{j}=1$ for $1 \leq j \leq n-1, C_{i} C_{j}=0$ 
when $|i-j|>1$. We have $C_{n-1} Z_{n}=1$ and the intersection point $p=C_{n-1} \cap \operatorname{Supp}\left(Z_{n}\right)$ is a non-singular point of $Z_{n}$. Since $\omega_{Z_{n}} \simeq \mathcal{O}_{Z_{n}}$, we have $\mathcal{O}_{Z_{n}}\left(K_{X}\right) \simeq \mathcal{O}_{Z_{n}}\left(-Z_{n}\right) \simeq$ $\mathcal{O}_{Z_{n}}\left(-Z_{n-1}+C_{n-1}\right)$. Therefore, $\mathcal{O}_{Z_{n}}(p) \simeq \mathcal{O}_{Z_{n}}\left(K_{X}+Z_{n-1}\right)$.

Note that we have $Z_{i}^{2}=-1$ and $p_{a}\left(Z_{i}\right)=1$ for any $i, 0 \leq i \leq n$. We know that the restriction map $H^{0}(X, L) \rightarrow H^{0}(Z, L)$ is surjective and that $h^{0}\left(Z_{i}, L\right)=1$ for each $i$ with $0 \leq i \leq n$, since $L$ is numerically equivalent to $K_{X}$. Consider the cohomology long exact sequence for

$$
0 \rightarrow \mathcal{O}_{C_{0}+\cdots+C_{n-1}}\left(L-Z_{n}\right) \rightarrow \mathcal{O}_{Z}(L) \rightarrow \mathcal{O}_{Z_{n}}(L) \rightarrow 0 .
$$

We have $H^{0}\left(C_{0}+\cdots+C_{n-1}, L-Z_{n}\right)=0$, since $L-Z_{n}$ is anti-nef of degree -1 on $C_{0}+\cdots+C_{n-1}$. Hence the restriction map $H^{0}(Z, L) \rightarrow H^{0}\left(Z_{n}, L\right)$ is an isomorphism. Let $s \in H^{0}(Z, L)$ be a non-zero element. Then $\left.s\right|_{Z_{n}}$ vanishes exactly at one point $q \in Z_{n}$ which is a non-singular point of $Z_{n}$, because $Z_{n}$ is numerically 2-connected. We have $\mathcal{O}_{Z_{n}}(q) \simeq \mathcal{O}_{Z_{n}}(L)$. Note that $s$ is constant on $C_{0}+\cdots+C_{n-1}$. It follows that $s \in H^{0}(Z, L)$, which vanishes at $q$, also vanishes on $C_{0}+\cdots+C_{n-1}$ if and only if $p=q$. By what shown above, we have $p=q$ if and only if $\mathcal{O}_{Z_{n}}(L) \simeq \mathcal{O}_{Z_{n}}\left(K_{X}+Z_{n-1}\right)$.

When $L=K_{X}$ on $Z_{n}$, the condition $\mathcal{O}_{Z_{n}}(L) \simeq \mathcal{O}_{Z_{n}}\left(K_{X}+Z_{n-1}\right)$ becomes $\mathcal{O}_{Z_{n}}\left(Z_{n-1}\right) \simeq \mathcal{O}_{Z_{n}}$ which is equivalent to $p_{g}\left(V_{n-1}, p_{n-1}\right)=2$ by $[9,2.20]$, where $\left(V_{n-1}, p_{n-1}\right)$ denotes the singularity obtained by contracting $Z_{n-1}$, and then $\left(V_{n-1}, p_{n-1}\right)$ is Gorenstein by $[9,3.5]$. We have $\operatorname{mult}\left(V_{n-1}, p_{n-1}\right)=2$ and $\operatorname{embdim}\left(V_{n-1}, p_{n-1}\right)=3$ by $[9,5.4]$.

Corollary 4.6. Let $(V, o)$ be an elliptic numerically Gorenstein singularity with $p_{g}(V, o) \geq 2$ whose fundamental cycle satisfies $Z^{2}=-1$ on the minimal resolution $X$. Then the fixed part of $\left|K_{X}\right|$ supports the exceptional set of the rational double point of type $\mathbf{A}_{n}$ if and only if the length of the elliptic sequence is $n+1$ and $p_{g}\left(V_{n-1}, p_{n-1}\right)=2$ holds for the singularity obtained by contracting $Z_{n-1}$, where $\left\{Z_{i}\right\}_{i=0}^{n}$ denotes the elliptic sequence.

EXAMPLE 4.7. Let $n$ be a positive integer and consider two hypersurface singularities respectively defined by the following equations:

$$
\text { (I) } z^{2}=x\left(x^{4 n+2}+y^{4}\right), \quad \text { (II) } z^{2}=x^{6(2 n+1)}+y^{3}
$$

Both are elliptic singularities and have the same minimal resolution dual graph as in Fig. 1 with $m=2 n$ and $\Delta_{m}$ being a (-1)-elliptic curve. We have $p_{g}(V, o)=n+1$ for (I) and $p_{g}(V, o)=2 n+1$ for (II). It can be checked directly that $\mathrm{Bs}\left|K_{X}\right|$ is one point when (I) is the case, while it consists of $2 n(-2)$-curves forming the Dynkin diagram of type $\mathbf{A}_{2 n}$ when (II).

5. Appendix (Isolated base points). We have ignored isolated base points so far. We can say at least the following about them. Although this is very similar to Theorem 1.1 and Proposition 2.2, we give a proof for the readers' convenience.

Proposition 5.1. Let $(V, o)$ be a normal surface singularity and $\pi: X \rightarrow V$ the minimal resolution. Let $L$ be a line bundle on $X$ such that $L-K_{X}$ is nef. If $x \in \mathrm{Bs}|L|$, then there exists a subcurve $\Delta$ of $Z$ satisfying:

(1) The restriction $H^{0}(Z, L) \rightarrow H^{0}(\Delta, L)$ is surjective.

(2) $\Delta$ is the fundamental cycle on its support, $\Delta^{2}=-1$ and $x$ is a non-singular point of $\Delta$. 
(3) $L-K_{X}$ is numerically trivial on $\Delta$ and $\mathcal{O}_{\Delta}(L) \simeq \omega_{\Delta} \otimes \mathcal{O}_{\Delta}(x)$.

Proof. We take an irreducible component $A$ through $x$ and let $\Delta=\Delta(A)$ be a minimal curve such that $A \preceq \Delta \preceq Z$ and the restriction map $H^{0}(Z, L) \rightarrow H^{0}(\Delta, L)$ is surjective. We see that $K_{\Delta}-L=\Delta-\left(L-K_{X}\right)$ is not nef on $\Delta$, since $L-K_{X}$ is nef and $\Delta^{2}<0$. It follows from [4, Lemma 2.2.1] that $A$ is of multiplicity one in $\Delta$, $K_{\Delta}-L$ is nef on $\Delta-A$ and the image of $H^{0}(Z, L) \rightarrow H^{0}(A, L)$ contains the image of the injection $H^{0}(A, L-(\Delta-A)) \rightarrow H^{0}(A, L)$. Since $K_{\Delta}-L$ is not nef on $\Delta$, we have $\left.\operatorname{deg}\left(L-K_{\Delta}\right)\right|_{A}=\left.\operatorname{deg}(L-(\Delta-A))\right|_{A}-\operatorname{deg} K_{A}>0$. From this, we infer that $H^{0}(\Delta, L) \rightarrow H^{0}(\Delta-A, L)$ is surjective. This allows us to assume that there are no components of $\Delta-A$ containing $x$. In fact, if there is another component $B \prec \Delta$ with $x \in B$, then we can argue with $B$ instead of $A$, since what we have shown implies that $\Delta(B) \preceq \Delta-A \prec \Delta=\Delta(A)$.

Then, since $x \in \mathrm{Bs}|L|$, we must have $x \in \operatorname{Bs}\left|\mathcal{O}_{A}(L-(\Delta-A))\right|$. Since $\operatorname{deg} \mathcal{O}_{A}(L-$ $(\Delta-A))>\operatorname{deg} K_{A}$, we see that $x$ is a non-singular point of $A$ and $\mathcal{O}_{A}(L-(\Delta-A)) \simeq$ $\mathcal{O}_{A}\left(K_{A}+x\right)$ (see, e.g., [10, p. 113]). The last isomorphism shows that $A \Delta+1=$ $\left.\operatorname{deg}\left(L-K_{X}\right)\right|_{A} \geq 0$. Then $0>\Delta^{2}=(\Delta-A) \Delta+A \Delta \geq\left.\operatorname{deg}\left(L-K_{X}\right)\right|_{\Delta-A}-1 \geq-1$. In sum, we get $A \Delta=\Delta^{2}=-1$ and see that $\mathcal{O}_{\Delta}\left(L-K_{X}\right)$ and $\mathcal{O}_{\Delta}(\Delta+x)$ are both numerically trivial. Since $H^{0}(\Delta, L) \rightarrow \mathcal{O}_{x}$ is zero, we have $H^{1}(\Delta, L-x) \neq 0$ which is equivalent to $H^{0}\left(\Delta, K_{\Delta}-L+x\right) \neq 0$. Since $\Delta^{2}=-1, \Delta$ is numerically 1-connected by Lemma 2.1. Since $\mathcal{O}_{\Delta}\left(K_{\Delta}-L+x\right)$ is numerically trivial, we conclude that $\mathcal{O}_{\Delta}(L) \simeq \mathcal{O}_{\Delta}\left(K_{\Delta}+x\right)$. We know that $\Delta$ is the fundamental cycle on its support, because $\Delta$ is numerically 1 -connected and $\mathcal{O}_{\Delta}(-\Delta)$ is nef.

LEMMA 5.2. Let the notation and assumptions be as above. Assume furthermore that $x$ is an isolated base point of $|L|$. If $p_{a}(\Delta)=1$, then $\Delta$ is numerically 2 -connected.

Proof. Recall that $\Delta$ is a numerically 1-connected curve that is the fundamental cycle on its support. If $\Delta$ is not 2-connected, then we have an effective decomposition $\Delta=\Delta_{1}+\Delta_{2}, \Delta_{1} \Delta_{2}=1$. In particular, $\Delta_{1}$ and $\Delta_{2}$ are both numerically 1-connected. Since $\Delta^{2}=-1$ and $p_{a}(\Delta)=1$, we have $K_{X} \Delta=1$ which enables us to assume that $K_{X} \Delta_{1}=1, K_{X} \Delta_{2}=0$. Since $\Delta_{1}^{2}+\Delta_{2}^{2}=-3$, we have $\Delta_{1}^{2}=-1$ and $\Delta_{2}^{2}=-2$ for the reason of parity. Hence $\Delta_{2}$ is a connected curve consisting of (-2)-curves, all with multiplicity one. Recall that $L$ and $K_{X}$ are numerically equivalent on $\Delta$ by Proposition 5.1. It follows that we have $\mathcal{O}_{\Delta_{2}}(L) \simeq \mathcal{O}_{\Delta_{2}}$. This implies that $x \notin$ $\operatorname{Supp}\left(\Delta_{2}\right)$, because $x$ is an isolated base point of $|L|$. Then $\mathcal{O}_{\Delta_{2}}(\Delta)$ is numerically trivial, because so is $\mathcal{O}_{\Delta}(\Delta+x)$. Hence $0=\Delta_{2} \Delta=\Delta_{2}\left(\Delta_{1}+\Delta_{2}\right)=1-2=-1$, a contradiction. Therefore, $\Delta$ is numerically 2 -connected. $\square$

We obtain an elliptic Gorenstein singularity with $p_{g}=1$ by contracting $\Delta$ as above. So, Lemma 5.2 gives us a clearer picture of $\mathrm{Bs}|L|$, when $(V, o)$ is an elliptic singularity and $L-K_{X}$ is nef: $\mathrm{Bs}|L|$ consists of $(-2)$-curves forming configurations of type $\mathbf{A}$ and several isolated points lying on the fundamental cycles (with selfintersection numbers -1 ) of elliptic Gorenstein singularities with $p_{g}=1$.

\section{REFERENCES}

[1] M. ARTin, Some numerical criteria for contractibility of curves on algebraic surfaces, Amer. J. Math., 84 (1962), pp. 485-496.

[2] M. Artin, On isolated rational singularities of surfaces, Amer. J. Math., 88 (1966), pp. 129136. 
[3] C. Ciliberto, P. Francia and M. Mendes Lopes, Remarks on the bicanonical map for surfaces of general type, Math. Z., 224 (1997), pp. 137-166.

[4] K. Konno, 1-2-3 theorem for curves on algebraic surface, J. reine. angew. Math., 533 (2001), pp. 171-205.

[5] H. B. Laufer, On minimally elliptic singularities, Amer. J. Math., 99 (1977), pp. 1257-1295.

[6] H. B. LAUFER, Weak simultaneous resolution for deformations of Gorenstein surface singularities, Proc. Symp. Pure Math., 40 (1983), pp. 1-29.

[7] H. B. Laufer, Generation of 4-pluricanonical forms for surface singularities, Amer. J. Math., 109 (1987), pp. 571-589.

[8] M. Mendes Lopes, Adjoint systems on surfaces, Bollettino U.M.I.(7), 10-A (1996), pp. 169179.

[9] A. NÉmethi, "Weakly" elliptic Gorenstein singularities of surfaces, Invent. Math., 137 (1999), pp. $145-167$.

[10] M. ReID, Chapters on Algebraic Surfaces, Complex algebraic geometry (Park City, UT, 1993), pp. 3-159, IAS/Park City Math. Ser., 3, Amer. Math. Soc., Providence, RI, 1997.

[11] Рh. Wagreich, Elliptic singularities of surfaces, Amer. J. Math., 92 (1970), pp. 419-454.

[12] S. S. T. YAU, Hypersurface dual graphs of normal singularities of surfaces, Amer. J. Math., 101 (1979), pp. 781-812.

[13] S. S. T. YAU, On maximally elliptic singularities, Transact. AMS 257, no.2 (1980), pp. 269-329. 